\title{
Caso 2/06 - Insuficiência Cardíaca na Evolução Tardia Depois de Infarto do Miocárdio em Mulher de 33 Anos de Idade
}

\author{
Case 2/06 - Heart failure in a 33 year-old woman in the late follow-up after \\ myocardial infarction
}

Otavio Celeste Mangili, Paulo J. Moffa, Luiz Alberto Benvenuti Instituto do Coração do Hospital das Clínicas - FMUSP - São Paulo, SP

Mulher de 33 anos de idade procurou atendimento médico em razão de piora da falta de ar há duas semanas.

A paciente sofreu, aos 31 anos de idade, infarto do miocárdio. Depois do infarto, evoluiu com dispnéia desencadeada por esforços moderados que progrediu até ser desencadeada por mínimos esforços e ocorrer mesmo em repouso. A paciente era tabagista.

O cateterismo cardíaco realizado dois meses após o infarto revelou pressões na aorta 120/80 mmHg e no ventrículo esquerdo (VE) 120/20 mmHg. A cineangiocoronariografia revelou lesão de $60 \%$ na origem do ramo interventricular anterior da artéria coronária esquerda. A ventriculografia revelou aumento de VE com acinesia ântero-lateral e apical, e insuficiência mitral moderada.

O exame físico no primeiro atendimento no Hospital, há um ano, revelou freqüência cardíaca de 70 bpm, pressão arterial 110/70 mmHg. 0 exame dos pulmões foi normal. 0 exame do coração revelou segunda bulha hiperfonética em área pulmonar e sopro sistólico $++/ 4$ em área mitral. 0 exame do abdome foi normal e não havia edema de membros.

O eletrocardiograma de há um ano revelou ritmo sinusal, freqüência 79 bpm, duração de QRS de 129 mseg, SÂQRS $-30^{\circ}$ para trás, ondas $\mathrm{Q}$ em $\mathrm{I}, \mathrm{aVL}, \mathrm{V}_{3}$ e $\mathrm{V}_{4}$, ondas $\mathrm{S}$ profundas em $V_{2}$ e $V_{3}$ e persistência de supradesnível de segmento ST em derivações precordiais. Os diagnósticos eletrocardiográficos foram de sobrecarga atrial esquerda, distúrbio intraventricular da condução do estímulo do tipo bloqueio de ramo esquerdo, bloqueio da divisão ânterosuperior do ramo esquerdo e áreas eletricamente inativas em paredes lateral e anterior (Fig. 1).

A radiografia de tórax revelou aumento da área cardíaca, sem sinais de congestão pulmonar.

Os exames laboratoriais naquela ocasião revelaram $12,6 \mathrm{~g} / \mathrm{dl}$ de hemoglobina, hematócrito 37\%, colesterol

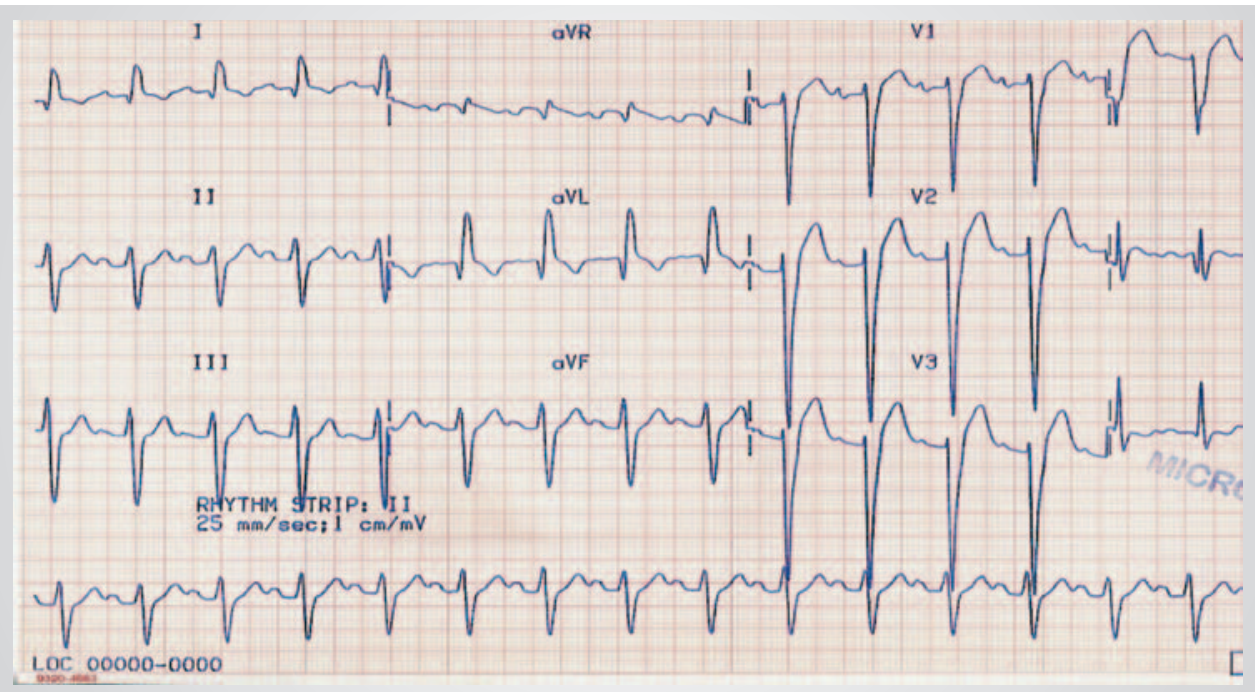

Fig. 1 - Eletrocardiograma - Sobrecarga atrial esquerda, distúrbio de condução intraventricular do estímulo, bloqueio da divisão ântero-superior do ramo esquerdo e área eletricamente inativa ântero-lateral 
$140 \mathrm{mg} / \mathrm{dl}$, triglicérides $64 \mathrm{mg} / \mathrm{dl}$, glicemia $107 \mathrm{mg} / \mathrm{dl} \mathrm{e}$ creatininemia $0,7 \mathrm{mg} / \mathrm{dl}$.

A avaliação por ecocardiograma, há um ano, revelou espessura diastólica do septo interventricular $9 \mathrm{~mm}$, da parede posterior VE $10 \mathrm{~mm}$, diâmetro diastólico de VE 82 mm, fração de ejeção 15\%, diâmetro da aorta 36 mm e do átrio esquerdo $61 \mathrm{~mm}$. As dimensões do ventrículo e do átrio direitos foram consideradas normais. Foram diagnosticadas discinesia apical, hipocinesia difusa do VE e insuficiência mitral acentuada.

Foi indicado o tratamento medicamentoso com 0,25 $\mathrm{mg}$ de digoxina, $40 \mathrm{mg}$ de furosemida, $5 \mathrm{mg}$ de cilazapril e $60 \mathrm{mg}$ de monitrato de isossorbida diários. Além do tratamento medicamentoso, foi indicado o tratamento cirúrgico eletivo - aneurismectomia, reconstrução geométrica do VE e tratamento cirúrgico da insuficiência da valva mitral.

Alguns meses depois, a paciente apresentou não só piora acentuada da dispnéia, que aparecia mesmo no repouso, com ortopnéia como também aparecimento de edema de membros inferiores, e necessitou internação para tratamento. Houve melhora dos sintomas e a paciente recebeu alta com prescrição de tratamento medicamentoso com $80 \mathrm{mg}$ de furosemida, $37,5 \mathrm{mg}$ de captopril, 0,25 mg de digoxina e $200 \mathrm{mg}$ de ácido acetilsalicílico diários.

O ecocardiograma contemporâneo a essa piora clínica revelou espessura distólica do septo interventricular 7 $\mathrm{mm}$, da parede posterior do VE $8 \mathrm{~mm}$, do VE $81 \mathrm{~mm}$ (diástole) e $69 \mathrm{~mm}$ (sístole). A fração de ejeção do VE foi de 38\%. O diâmetro da aorta foi $35 \mathrm{~mm}$ e do átrio esquerdo $50 \mathrm{~mm}$; o ventrículo e o átrio direitos estavam dilatados. Havia hipocinesia acentuada e difusa do VE e acentuada insuficiência mitral.

A paciente evoluiu bem por um mês e houve piora acentuada da dispnéia e aparecimento de dor torácica. Foi novamente internada para controle dos sintomas.
O exame físico na última internação revelou freqüência cardíaca 96 bpm, pressão arterial audível em torno de $80 \mathrm{mmHg}$. 0 exame dos pulmões foi normal. 0 exame do coração revelou sopro sistólico em área mitral $++/ 4$; não havia alterações no exame do abdome e dos membros inferiores.

O tratamento medicamentoso incluiu a administração de dobutamina e furosemida por via venosa, além de $37,5 \mathrm{mg}$ de captopril e $40 \mathrm{mg}$ de enoxaparina diários por via subcutânea.

O eletrocardiograma revelou ritmo sinusal, freqüência 100 bpm, bloqueio atrioventricular de primeiro grau, sobrecarga atrial esquerda, distúrbio de condução intraventricular, bloqueio da divisão ântero-superior do ramo esquerdo e área inativa anterior extensa (Fig. 2).

A evolução laboratorial revelou progressiva elevação da creatinina que passou de 1,2 mg/dl para 3,2 mg/dl; a uréia passou de $41 \mathrm{mg} / \mathrm{dl}$ para $125 \mathrm{mg} / \mathrm{dl}$; as enzimas hepáticas AST e ALT passaram de 21 e $10 \mathrm{UI} / \mathrm{I}$ para 526 e 522 UI/I. A taxa de leucócitos elevou-se de 6900/. $\mathrm{mm}^{3}$ para $13900 / \mathrm{mm}^{3}$, e instalou-se progressiva plaquetopenia de $223000 / \mathrm{mm}^{3}$ para $95000 / \mathrm{mm}^{3}$. Houve também alterações nos testes de coagulação. O INR passou de 1,36 para 3,67 e relação de tempos de TTPA de 1 para 2,91 . Houve estabilidade das dosagens séricas do sódio e do potássio: $140 \mathrm{mEq} / / \mathrm{l}$ para $150 \mathrm{mEq} / \mathrm{l}$ de sódio, e de 5,1 $\mathrm{mEq} / \mathrm{l}$ de potássio para 4,3 mEq/l. Havia hipoproteinemia - proteínas totais de 5,7 g/dl e albumina de 3,3 g/dl.

Apesar do tratamento administrado, a hipoxemia foi progressiva e a paciente necessitou intubação orotraqueal para suporte respiratório no quinto dia da internação.

A avaliação por cintilografia de perfusão pulmonar revelou hipoperfusão de bases de ambos os pulmões. As alterações foram consideradas de baixa probabilidade para tromboembolia pulmonar.

As medidas obtidas por meio da monitorização hemodinâmica com o emprego do cateter de Swan-Ganz

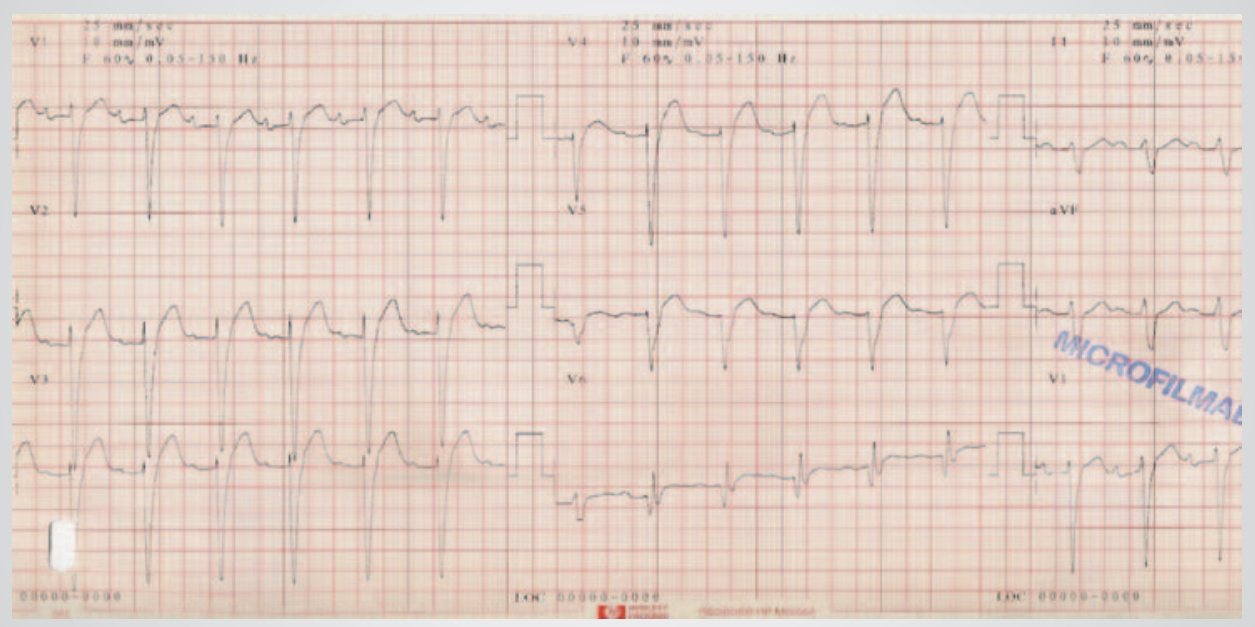

Fig. 2 - Eletrocardiograma - Bloqueio atrioventricular de primeiro grau, sobrecarga atrial esquerda, distúrbio de condução intraventricular, bloqueio da divisão ântero-superior do ramo esquerdo e área inativa anterior extensa 
demonstraram baixo débito cardíaco, hipotensão arterial, aumento de resistência vascular sistêmica e hipertensão arterial e capilar pulmonar. Na evolução houve queda da resistência sistêmica, sem alteração da circulação pulmonar (Tab. 1).

\begin{tabular}{|c|c|c|}
\hline \multicolumn{3}{|c|}{$\begin{array}{c}\text { Tabela } 1 \text { - Variáveis obtidas na monitorização } \\
\text { hemodinâmica à beira do leito }\end{array}$} \\
\hline & $3^{\circ}$ dia internação & $7^{\circ}$ dia internação \\
\hline $\begin{array}{l}\text { Drogas vasoativas } \\
\qquad(\mu \mathrm{g} / \mathrm{kg} / \mathrm{min})\end{array}$ & $\begin{array}{l}\text { Dobutamina } \\
20 \mu \mathrm{g} / \mathrm{kg} / \mathrm{min} ; \\
\text { Noradrenalina } \\
24 \mu \mathrm{g} / \mathrm{kg} / \mathrm{min}\end{array}$ & $\begin{array}{l}\text { Dobutamina } \\
20 \mu \mathrm{g} / \mathrm{kg} / \mathrm{min} ; \\
\text { Noradrenalina } \\
65 \mu \mathrm{g} / \mathrm{kg} / \mathrm{min}\end{array}$ \\
\hline Temperatura central $\left({ }^{\circ} \mathrm{C}\right)$ & 38,1 & 40,1 \\
\hline Freqüência cardíaca (bpm) & 99 & 133 \\
\hline Átrio Direito $(\mathrm{mmHg})$ & 12 & 13 \\
\hline \multicolumn{3}{|l|}{$\begin{array}{l}\text { Pressões em artéria } \\
\text { pulmonar }(\mathrm{mmHg})\end{array}$} \\
\hline Sistólica/ diastólica/ média & $67 / 56 / 62$ & $63 / 57 / 59$ \\
\hline Pressão de oclusão pulmonar & 46 & 36 \\
\hline \multicolumn{3}{|l|}{$\begin{array}{l}\text { Pressão arterial } \\
\text { sistêmica }(\mathrm{mmHg})\end{array}$} \\
\hline Índice cardíaco (I/min/kg) & 1,28 & 2,13 \\
\hline \multicolumn{3}{|l|}{$\begin{array}{l}\text { Resistência vascular } \\
\text { (dyn.seg.cm } \text { cm }^{-5} \text { ) }\end{array}$} \\
\hline Pulmonar & 711 & 518 \\
\hline Sistêmica & 2310 & 946 \\
\hline
\end{tabular}

A paciente apresentou taquicardia ventricular sustentada, revertida com cardioversão elétrica de 200 $\mathrm{J}$ no quinto dia de internação.

A evolução do quadro deu-se com choque e hipertermia persistentes. Foram acrescentados ceftriaxone $2 \mathrm{~g}$ e clindamicina 2400 mg diários. Na evolução apresentou parada cardiorrespiratória refratária às manobras de ressuscitação e faleceu no sétimo dia da internação.

Interpretação do eletrocardiograma - O traçado inicial não deve ser considerado como bloqueio de ramo esquerdo e sim como distúrbio de condução periinfarto, por isso pode ser feito o diagnóstico de área eletricamente inativa anterior extensa (Fig. 1).

O segundo traçado (Fig. 2) revela ritmo sinusal, freqüência cardíaca de 100 bpm, duração do intervalo PR de 180 ms, do intervalo QRS 130 ms, do intervalo QT 300 ms. O eixo elétrico do QRS está localizado a $60^{\circ}$ para trás. Podemos observar alterações na duração da onda $\mathrm{P}$, e fase negativa lenta em V1, presença de onda $r$ de V1 a V4 interior a $3 \mathrm{~mm}$, onda q patológica em V5. Verifica-se também onda S em V2 e V3 de 35 $\mathrm{mm}$. A onda $T$ demonstra morfologia sugestiva de ação digitálica, e é negativa em I, aVL, e V4, V5, e V6. Os achados configuram sobrecarga atrial esquerda, bloqueio divisional ântero-superior do ramo esquerdo do feixe de His, área eletricamente inativa em derivações da parede anterior ventricular.

(Dr. Paulo J. Moffa)

\section{Aspectos CLÍNICOS}

A presente paciente relatou infarto agudo do miocárdio (IAM) no início da $4^{a}$ década de vida, que se faz corroborar pela presença da onda $Q$ em DI, aVL, V3 e V4 e persistência de supradesnível de ST o que sugere formação de aneurisma, e acinesia ântero-lateral e apical de VE em cineangiocoronariografia e discinesia apical em ecocardiograma, todos sugestivos de infarto em topografia do ramo interventricular anterior da coronária esquerda.

O IAM em pacientes adultos com menos de 45 anos é um fenômeno incomum. Estudos das décadas de 1970 e 1980 sugeriam que entre 2 e $6 \%$ dos infartos ocorriam em pacientes jovens. Séries de 1990s sugerem que entre 4 e $10 \%$ dos infartos incidiam nessa faixa etária ${ }^{1}$. Além disso, estudo recente evidenciou que mulheres correspondem a $20 \%$ dos IAM abaixo de 45 anos de idade e 55\% acima de 75 anos $^{2}$.

Existem vários fatores de risco para IAM nos adultos jovens. A a maioria está relacionada à aterosclerose $(80 \%$ dos casos). Porém, IAM na ausência de aterosclerose, embora mais raro em faixas etárias maiores, corresponde a $20 \%$ dos casos abaixo de 45 anos; $5 \%$ são atribuídos a êmbolo coronário e outros $5 \%$ a estados de hipercoagulabilidade ${ }^{1}$.

Para os casos ligados à aterosclerose, existem tanto fatores de risco convencionais quanto novos fatores de risco. Merecem consideração na população jovem², entre os fatores de risco coronário clássicos, o tabagismo, a dislipidemia, a história familiar, e, em menor freqüência, a hipertensão arterial, o diabetes mellitus; nessa faixa etária entram, ainda, como fatores de risco, o uso de cocaína e as síndromes trombofílicas ${ }^{3,4}$.

O tabagismo parece ser o principal fator de risco para IAM em jovens. Entre 76 e $91 \%$ dos pacientes jovens com IAM são fumantes, comparados com $40 \%$ dos pacientes mais idosos.

A dislipidemia está presente entre $12 \%$ e $89 \%$ dos jovens com IAM. A hipercolesterolemia familiar homozigótica parece ter relação mais consistente com aterosclerose prematura. Porém aumentos de triglicérides e queda de HDL-colesterol também foram associados a IAM abaixo de 45 anos.

A prevalência de história familiar positiva entre familiares de primeiro grau de jovens infartados varia de 14 a $69 \%$, constituindo um fator de risco maior. Estudos em gêmeos demonstraram que o risco relativo de óbito em homens cujo irmão morreu de DAC antes de 55 anos é de 8,0 .

A hipertensão é menos comum em jovens com IAM que em idosos, tal como ocorre com o diabetes mellitus que também está mais relacionado ao IAM em idosos que em jovens, e menos de $10 \%$ destes últimos são diabéticos.

A cocaína é reconhecida como fator de risco para IAM em jovens, particularmente na forma de crack. 
Agudamente, a cocaína aumenta a freqüência cardíaca e a pressão arterial e diminui o fluxo sangüíneo coronariano pela indução de vasoespasmo, levando a aumento na demanda e à redução no aporte de oxigênio. 0 uso crônico de cocaína pode levar a aumento no processo de aterosclerose. Além desses, a droga pode levar à necrose miocárdica focal por miocardiotoxicidade.

Várias anormalidades do sistema de coagulaçâo são descritas como associadas ao IAM. O European Concerted Action on Thrombosis (ECAT) ${ }^{5}$ seguiu 2960 pacientes com angina de peito por 2 anos. De 106 pacientes com evento coronário, $12 \%$ estavam com menos que 45 anos e o nível sérico de fibrinogênio, fator de $\checkmark W B$ e tPA, foram preditores independentes de eventos coronarianos agudos. A atividade fibrinolítica diminuída com a resultante de níveis aumentados de PAI-1 também foi descrita em jovens com IAM. O uso de contraceptivos orais, que produzem estado pró-trombótico, tem sido considerado importante fator de risco para IAM, embora recentemente tenha decrescido de importância devido à diminuição de concentração hormonal. Deficiências de proteínas anticoagulantes, como proteína $\mathrm{C}$, proteína $\mathrm{S}$ e antittrombina III são mais relacionadas a trombose venosa. A presença de fator $V$ de Leiden não foi associada a evento trombótico arterial em nenhuma idade.

Dados do Coronary Artery Surgery Registry ${ }^{4}$ mostram que os 504 pacientes com menos de 45 anos tiveram com maior freqüência coronariopatia não obstrutiva e doença de um único vaso. Klein e cols. ${ }^{6}$ descreveram prevalência de doença coronária em 85 pacientes (59 após infarto) e encontraram doença de um vaso em $51 \%$, dois vasos em $31 \%$ e em três vasos em $19 \%$

A paciente evoluiu com dilatação e disfunção ventricular sistólica acentuadas e insuficiência mitral secundária. Alguns reparos devem ser feitos em relação ao tratamento medicamentoso inicial. Apesar de dose otimizada de inibidor de ECA, não houve relato de uso de betabloqueador, cujos benefícios tanto na prevenção da morte por arritmia quanto na evolução da falência miocárdica foram evidenciados nos estudos USCarvedilol $^{7}$, CIBIS $\|^{8}$ e MERIt-HF ${ }^{9}$. Cabe ainda ressaltar, como evidenciado no estudo CARMEN ${ }^{10}$, que o papel dos betabloqueadores no remodelamento ventricular e na melhora de fração de ejeção, além de mais expressivos, são aditivos aos inibidores da ECA.

O tratamento inicial da paciente não incluía antiplaquetários. Está bem demonstrado que o tratamento com antiplaquetários foi benéfico para pacientes que sofreram infarto do miocárdio e para portadores de cardiopatia isquêmica crônica por aterosclerose coronária ${ }^{11}$.

Além disso, embora não haja relato de dor precordial durante seguimento ambulatorial, fazia uso de nitratos, medicação para qual não foi demonstrada influência na taxa de sobrevivência de portadores de coronariopatia crônica.

Houve indicação de terapia cirúrgica, aneurismectomia e reconstrução geométrica de $V E$, além de tratamento cirúrgico da valva mitral. Os aneurismas ventriculares são graves complicações do infarto do miocárdio transmural, com importante repercussão clínica (insuficiência cardíaca, tromboembolismo, angina e arritmias); ocorrem mais freqüentemente em obstruções proximais da artéria descendente anterior. 0 tratamento cirúrgico tem sido indicado freqüentemente, com melhora de sintomas, qualidade de vida e sobrevida dos pacientes. A insuficiência cardíaca, tromboembolismo arterial sistêmico e arritmias ventriculares são as principais indicações de correção cirúrgica do aneurisma ${ }^{12}$.

A insuficiência mitral faz parte do cortejo evolutivo do remodelamento ventricular em pacientes com insuficiência cardíaca, principalmente por dilatação do anel valvar. A indicação do emprego da anuloplastia ou da substituição valvar mitral no tratamento de insuficiência cardíaca faz-se na presença de insuficiência mitral moderada a grave.

Devem ser considerados para este procedimento pacientes em CFIII ou IV, apesar de otimização do tratamento medicamentoso. O emprego das técnicas cirúrgicas sobre o aparelho valvar mitral em cardiomiopatia dilatada, embora eficientes na melhora de qualidade de vida dos pacientes, não conferiam aumento de sobrevivência ${ }^{13,14}$

O quadro da paciente evoluiu com episódios de descompensação aguda da $0=$ insuficiência cardíaca, tendo sido internada por duas ocasiões no intervalo de dois a três meses. Dentre as causas de descompensação aguda de insuficiência cardíaca vale ressaltar: redução inapropriada da medicação e abuso na dieta ${ }^{15,16}$; arritmias cardíacas, destacando-se a fibrilação atrial que leva à perda do impulso sistólico atrial, e as bradicardias por distúrbio na condução atrioventricular, ambas com queda de débito cardíaco; isquemia ou infarto do miocárdio; neste caso, um novo evento isquêmico não pode ser descartado, uma vez que houve rápida piora funcional e alteração evolutiva no eletrocardiograma; infecção sistêmica, que leva ao aumento do metabolismo corporal total e taquicardia; tromboembolia pulmonar ${ }^{17}$ (TEP); estados de alto débito; administração de depressores cardíacos ou drogas retentoras de sal. Embora a paciente tenha apresentado cintilografia de ventilação-perfusão pulmonar com baixa probabilidade de TEP, cabe ressaltar que ela apresentou como sintomas que motivaram internação: dispnéia acentuada e dor torácica que levantaram tal hipótese diagnóstica. Além do mais, a presença de TEP em paciente com insuficiência cardíaca é forte preditor de mortalidade em curto e longo prazos ${ }^{17}$.

Houve evolução do quadro de descompensação aguda, com necessidade de suporte inotrópico. Apesar de levarem à melhora de contratilidade cardíaca, o uso de inotrópicos como a dobutamina está associado a aumento de consumo de oxigênio pelo miocárdio e da incidência de arritmias, não havendo benefício claro em mortalidade.

Embora ainda não disponível para uso comercial na 
época, o levosimendan, em comparação com dobutamina, como evidenciado no estudo LIDO, em pacientes com índice cardíaco menor que 2,5 l/min, teve benefício imediato significativo ${ }^{18}$

Diante da evolução do quadro de descompensação aguda, revelada pela insuficiência renal aguda e agressão hepática, e das medidas hemodinâmicas iniciais terem corroborado o diagnóstico de choque cardiogênico, devese aventar a hipótese de se lançar mão de assistência circulatória mecânica. Os tipos de assistência circulatória mecânica podem ser divididos em: assistência em série por contrapulsação (balão intra-aórtico), assistência em paralelo (bombas de fluxo contínuo, ventrículos artificiais) e substituição mecânica total (coração artificial).

O implante de dispositivos de assistência circulatória como ponte para o transplante cardíaco está indicado em pacientes que apresentem choque de origem cardiogênica, refratário ao uso de terapia medicamentosa e ao controle adequado da volemia. São inclusos pacientes com diagnóstico de insuficiência cardíaca congestiva crônica descompensada ou de insuficiência cardíaca aguda, em condições clínicas e psicossociais presumivelmente aceitáveis para a indicação de transplante.

São critérios hemodinâmicos de indicação de assistência circulatória mecânica:

Falência do VE com índice cardíaco $<1,8$ l/min. $\mathrm{m}^{2}$, pressão arterial sistólica $<90 \mathrm{mmHg}$ e pressão oclusão pulmonar > 20 mmHg; falência de ventrículo direito:índice cardíaco $<1,8 \mathrm{l} / \mathrm{min} \cdot \mathrm{m}^{2}$, pressão de oclusão pulmonar $<10 \mathrm{mmHg}$, pessão venosa central $>20 \mathrm{mmHg}$.

Embora não tenha havido oportunidade de sua indicação, o transplante cardíaco é a terapia de escolha no tratamento dos pacientes portadores de insuficiência cardíaca avançada. A seleção dos candidatos, porém, deve ser criteriosa com identificação de fatores de mau prognóstico e enfermidades coexistentes, que poderão transformar-se em critérios de exclusão. Na avaliação clínica, deve-se confirmar a gravidade da cardiopatia, descartar a possibilidade de terapias alternativas, excluir contra-indicações, bem como estimar o prognóstico da doença em curto prazo.

São indicações absolutas ao transplante: consumo máximo de $02<10 \mathrm{ml} / \mathrm{kg}$.min, classe funcional IV da NYHA, hospitalizações recorrentes por insuficiência cardíaca, isquemia miocárdica limitante e não suscetível de revascularização, arritmias ventriculares sintomáticas recorrentes e refratárias ao tratamento clínico.

São indicações relativas ao transplante: consumo de 02 máximo $<14 \mathrm{ml} / \mathrm{kg} / \mathrm{min}$ e limitação importante na atividade diária, Classe II-IV, hospitalizações recorrentes por insuficiência cardíaca, arritmia ventricular freqüente, com antecedente de morte súbita ressuscitada.

Hipóteses diagnósticas - Aterosclerose coronária, infarto de parede anterior com formação de aneurisma de VE e posterior dilatação ventricular levando à miocardiopatia isquêmica, insuficiência cardíaca descompensada e choque cardiogênico. No evento final houve sobreposição de infecção com septicemia.

(Dr. Otavio Celeste Mangili)

\section{NeCROPSIA}

O coração pesou $600 \mathrm{~g}$, havendo acentuado aumento volumétrico. Cortes transversais demonstraram acentuada dilatação e hipertrofia excêntrica do VE, com cicatriz fibrosa de extenso infarto transmural prévio acometendo a parede anterior e parte das paredes lateral e septal do VE. A cicatriz fibrosa estendia-se da base ao ápice cardíaco, notando-se afilamento e discreta dilatação aneurismática da parede anterior (Fig. 3). Havia dilatação do anel da valva mitral, que não exibia lesões anatomopatológicas primárias. Não havia trombos cavitários. As artérias coronárias apresentavam dominância direita tendo sido dissecadas e analisadas histologicamente. Havia placa aterosclerótica excêntrica nos primeiros centímetros do ramo interventricular anterior (Fig. 4), com obstrução de $90 \%$ da luz no segundo centímetro. A placa apresentava

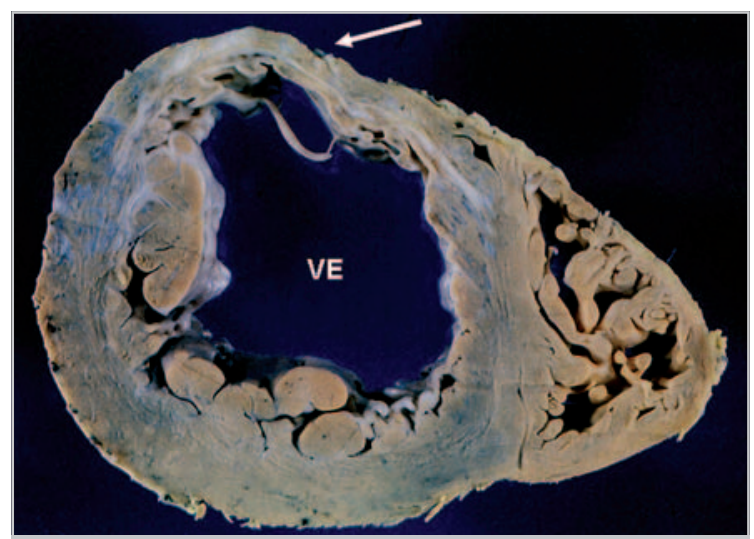

Fig. 3 - Corte transversal de ambos ventrículos exibindo cicatriz fibrosa de infarto transmural prévio, acometendo as paredes anterior, lateral e septal do ventrículo esquerdo, com afilamento e discreto abaulamento aneurismático da parede anterior (seta). Note a intensa dilatação da cavidade ventricular esquerda (VE)

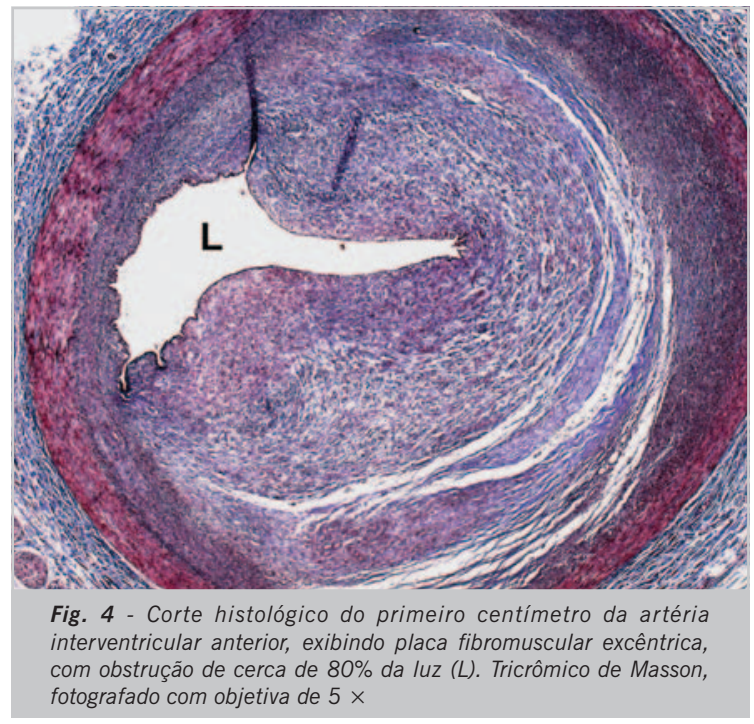


constituição fibromuscular, sendo desprovida de conteúdo lipídico (Fig. 5A), notando-se áreas focais de neovascularização, compatíveis com trombos organizados focais (Fig. 5B). Placas excêntricas menores, de constituição fibromuscular, estavam presentes no primeiro centímetro da artéria circunflexa, com obstrução de 30\% e no primeiro centímetro da artéria descendente posterior, com obstrução de $40 \%$. Os pulmões pesaram $1.310 \mathrm{~g}$ em conjunto, notando-se congestão passiva crônica, caracterizada por discreto espessamento da parede das veias e capilares pulmonares e pela presença de pigmento hemosiderótico em histiócitos localizados nos espaços alveolares. Havia bronquite crônica e enfisema pulmonar incipiente, com áreas focais de acúmulo de histiócitos albergando pigmento relacionado ao fumo (Fig. 6).

Encontrou-se broncopneumonia em ambas as bases pulmonares, o que constituiu a causa terminal do óbito. O fígado apresentava dilatação de sinusóides em região centrolobular, com áreas de necrose hemorrágica, secundária a insuficiência cardíaca congestiva e choque hemodinâmico. Presença de cicatriz de infarto esplênico.

(Dr. Luiz Alberto Benvenuti)

Diagnósticos anatomopatológicos - Aterosclerose coronariana, com placas fibromusculares e evidências de trombose antiga focal, organizada; cardiopatia isquêmica, com extenso infarto transmural cicatrizado da parede ântero-látero-septal do VE; alterações pulmonares secundárias a tabagismo crônico; broncopneumonia (causa terminal do óbito).

(Dr. Luiz Alberto Benvenuti)

\section{Comentários}

Trata-se de paciente jovem, do sexo feminino, com cardiopatia isquêmica descompensada, secundária a extenso infarto transmural prévio, cicatrizado, que atingiu a parede anterior e parte da lateral e septal do VE. A dilatação do VE era acentuada e global, com discreta proeminência da parede anterior, o que é compatível com resquício de aneurisma dessa parede. 0 estudo das artérias coronárias mostrou lesão isolada do segmento inicial da artéria interventricular anterior, com obstrução de 90\% da luz, caracterizada pela presença de placa fibromuscular excêntrica, desprovida de conteúdo lipídico e com áreas focais de trombose organizada. Havia evidências clínicas e anatomopatológicas de tabagismo crônico.

Nos países desenvolvidos, assim como entre os homens, a cardiopatia isquêmica é a principal causa de morte entre as mulheres. Além dos tradicionais fatores de risco, deve-se considerar nas mulheres os fatores protetores relacionados aos níveis de estrógeno, o que explica o aumento significativo na incidência da doença arterial coronária nas mulheres após a menopausa. Em mulheres jovens, o tabagismo, presente de forma indiscutível no caso em questão, é considerado o

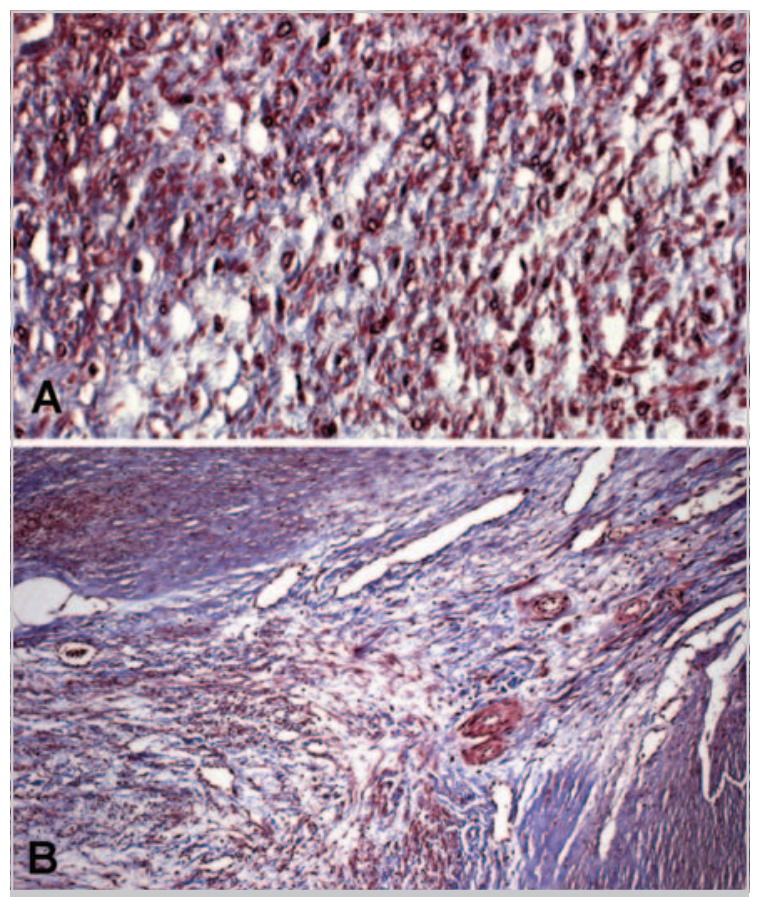

Fig. 5 - Detalhes histológicos das placas ateroscleróticas das artérias coronárias, evidenciando a constituição fibromuscular, destituída de conteúdo lipídico (A) e áreas de neovascularização, compatível com trombo organizado (B). Tricrômico de Masson, fotografado com objetiva de $40 \times(A)$ e de $10 \times(B)$

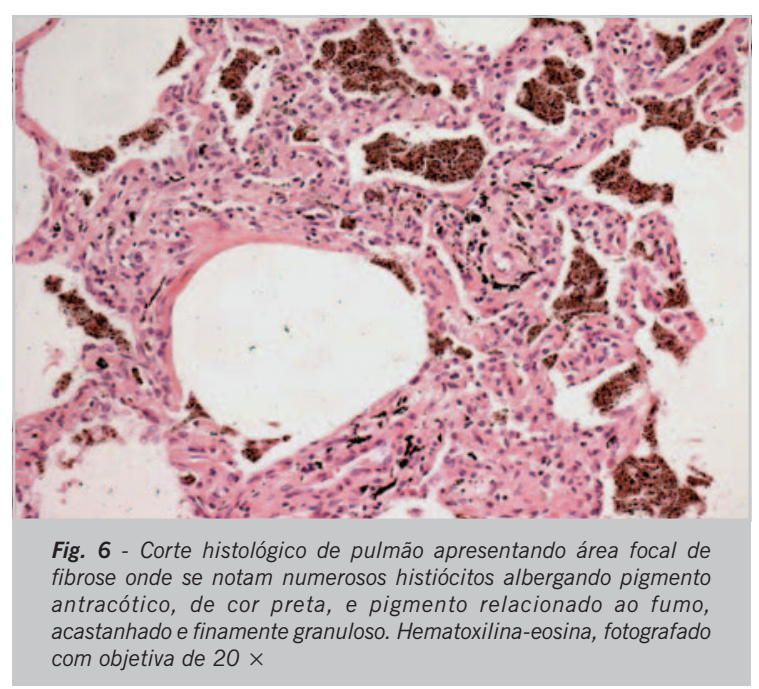

principal fator de risco da doença arterial coronária, e sua ação deletéria parece estar relacionada diretamente à quantidade de cigarros consumidos por dia ${ }^{19}$.

O tabaco apresenta efeito aterogênico devido à toxicidade endotelial, alterações do perfil lipídico e também ao efeito trombogênico. Pode ainda estar associado à ocorrência de espasmo arterial. O risco associado ao tabagismo aumenta consideravelmente na presença de outros fatores, particularmente hipertensão arterial sistêmica, diabetes mellitus e uso de anticoncepcionais orais. A presença de fatores trombofílicos, que facilitam a ocorrência de trombose vascular, é outro fator que deve 
ser investigado nos casos de doença arterial coronária em jovens. Estudo recente mostrou que esses fatores estiveram presentes em grande número de pacientes jovens com infarto agudo do miocárdio, de ambos os sexos, particularmente naqueles com poucos fatores de risco tradicionais ${ }^{20}$.

No presente caso, não temos informações sobre o uso de anticoncepcionais orais e sobre a ocorrência de outros fatores trombofílicos, que não o tabagismo. Interessante notar nesta paciente que a placa aterosclerótica da lesão coronariana era destituída de lipídios, e apresentava constituição fibromuscular, com áreas focais sugestivas de trombose organizada. A causa terminal do óbito foi broncopneumonia, ocorrida na evolução de paciente em grave choque cardiogênico.

(Dr. Luiz Alberto Benvenuti)

\section{REFERÊNCIAS}

1. Jalowiec DA, Hill J. Myocardial infarction in the young and women. Cardiovascular Clinics. 1989; 20:197-206.

2. Chen L, Chester M, Kaski JC. Clinical factors and angiographic features associated with premature coronary artery disease. Chest. 1995; 108:364-9.

3. Zimmerman FH, Cameron A, Fisher LD. Myocardial infarction in young adults: angiographic characterization, risk factors and prognosis. (Coronary Artery Surgery Registry). J Am Col Cardiol. 1995;26:654-61.

4. Haverskate F, Thompsom SG, Pyke SDM et al. for the European Concerted Action on Thrombosis and Disabilities Angina Pectoris Study Group. Production of C-reative protein and risk coronary events in stable and unstable angina. Lancet. 10997; 349:462-66.

5. Klein LW, Agarwal JB, Herlich MB et al. Prognosis of symptomatic coronary artery disease in young adults aged 40 years or less. Am $\mathrm{J}$ Cardiol. 1987; 60:1269-72.

6. Bristow MR, Gilbert EM, Abraham WT et al. Carvedilol produces dose-related improvements in left ventricular function and survival in subjects with chronic heart failure. Circulation. 1996; 94:2807-16.

7. The CIBIS-II investigators and commitees. The cardiac Insufficiency bisoprolol study II (CIBIS-II): a randomized trial. Lancet. 1999;353:9-13.

8. Merit-HF Study group. Effect of metoprolol CR/XL in chronic heart failure: Metoprolol $\mathrm{CR} / \mathrm{XL}$ randomized intervention trial in congestive heart failure (MERIT-HF). Lancet 1999; 353:2001-07.

9. Remme WJ, Riegger G, Hildebrandt $P$ et al. The benefits of early combination treatment of carvedilol and an ACE-inhibitor in mild heart failure and left ventricular systolic dysfunction. The carvedilol and ACEinhibitor remodelling mild heart failure evaluation trial (CARMEN). Cardiovasc Drugs Ther. 2004;18:57-66.

10. Juul-Moller S, Edvardsson N, Jahnmatz B et al. Double blind trial of

aspirin in primary prevention of myocardial infartion in patients with stable chronic angina pectoris. Lancet. 1992; 114:1421-5.

11. Da Silveira WL, Leite AF, Soares ECG et al. Short-term follow-up of patients after aneurysmectomy of left ventricle. Arq Bras Cardiol. 2000; 75:401-4

12. Bollling SF, Pagani FD, Deeb GM, Bach DS. Intermediate-term outcome of mitral recosntruction in cardiomyopathy. J Thorac Cardiovasc Surg. 1998; 15:381-8.

13. Calafiore AM, Gallina S, Di Mauro M, et al. Mitral valve procedure in dilated cardiomyopathy : repair or replacement. Ann Throrac Surg. 2001;71:1146-53.

14. Bennett SJ, Huster GA, Baker SL, et al. Characterization of the precipitants of hospitalization for heart failure decompensation. Am J Critical Care. 1998; 7:168-74.

15. Michalsen A, König G, Thimme W. Preventable causative factors leading to hospital admission with decompensated heart failure. Heart. 1998; 80:437-41.

16. Goldhaber SZ, Visani L, De Rosa M. Acute pulmonary embolism: Clinical outcomes in the International Cooperativa Pulmonary Embolism Registry. Lancet 1999; 353:1386-9.

17. Innes CA, Wagstaff AJ. Levosimendan: a review of its use in the management of acute decompensated heart failure. Drugs 2003; 63:2651-71.

18. Brezinka V, Padmos I. Coronary heart disease risk factors in women. Eur Heart J 1994; 15:1571-84.

19. Thomas D. Tobacco smoking and cardiovascular diseases. Rev Prat 1993; 43:1218-22.

20. Segev A, Ellis MH, Segev F et al. High prevalence of thrombophilia among young patients with myocardial infarction and few conventional risk factors. Int J Cardiol 2005; 98:421-424. 